\title{
A Nonmonotonous Damage Model to Characterize Mullins and Residual Strain Effects of Rubber Strings Subjected to Transverse Vibrations
}

\author{
Alex Elías-Zúñiga, Karen Baylón, Oscar Martínez-Romero, \\ Ciro A. Rodríguez, and Héctor R. Siller \\ Centro de Innovación en Diseño y Tecnología, Tecnoloógico de Monterrey, Campus Monterrey, E. Garza Sada 2501 Sur, \\ 64849 Monterrey, NL, Mexico
}

Correspondence should be addressed to Alex Elías-Zúñiga; aelias@itesm.mx

Received 27 May 2013; Revised 25 August 2013; Accepted 4 September 2013

Academic Editor: Jun Zhang

Copyright (C) 2013 Alex Elías-Zúñiga et al. This is an open access article distributed under the Creative Commons Attribution License, which permits unrestricted use, distribution, and reproduction in any medium, provided the original work is properly cited.

This work focuses on the formulation of a constitutive equation to predict Mullins and residual strain effects of buna- $\mathrm{N}$, silicone, and neoprene rubber strings subjected to small transverse vibrations. The nonmonotone behavior exhibited by experimental data is captured by the proposed material model through the inclusion of a phenomenological non-monotonous softening function that depends on the strain intensity between loading and unloading cycles. It is shown that theoretical predictions compare well with uniaxial experimental data collected from transverse vibration tests.

\section{Introduction}

It is well known that the response of rubber-like materials when subjected to cyclic loading, the load needed to stretch the material sample to a given stretch value during the second loading cycle in tension, compression, or shear deformation states, to say a few, is smaller than that required to reach the same stretch during the primary loading cycle. This reduction in the stress magnitude is known as the Mullins effect [1, 2]. To characterize this effect, there exist in the literature many different material models that have been developed to explain material damage mechanisms. See, for instance, the papers of Govindjee and Simo [3], Ogden and Roxburgh [4], Johnson and Beatty [5], Beatty and Krishnaswamy [6], Elías-Zúñiga and Beatty [7], Holzapfel et al. [8], de Souza Neto et al. [9], Elías-Zúñiga [10], Dorfmann and Ogden [11], Marckmann et al. [12], Diani et al. [13], KazakevičiūtèMakovska and Kačianauskas [14], de Tommasi et al. [15], Rickaby and Scott [16], Córdova et al. [17], and references cited therein for an overview of the main features of these models. Another effect that has been observed in rubberlike materials during the unloading process is related to the so-called healing effect in which a fraction of the broken cross-links can be reformed [18]. It is also known that the fraction percentage of reformed cross-links depends on the maximum and minimum strains of the loading cycles. Due to this healing effect, the material behavior upon unloading-reloading cycles is not elastic because of the hysteresis effects [19]. Other phenomena such as stressinduced anisotropy as well as crystallization in cyclic loading of polymeric-like materials are discussed in [13] and [20], respectively.

In the present work, we neglect healing and anisotropic material effects and use a constitutive material model to characterize softening and residual strain effects of rubberlike cords subjected to small transverse vibrations test. In particular, we characterize the nonmonotonous behavior shown by experimental data by using a nonmonotonous stresssoftening function that depends on the amount of strain intensity. To consider residual strain effects, the procedure developed by Holzapfel et al. in [8] is used to develop a strain energy density function which depends on a damage parameter variable that becomes active only during unloading conditions. We will show that the nonmonotonous 
damage function describes well experimental data when the normalized transverse frequency ratio is plotted against the normalized strain intensity ratio $[10,21]$.

The paper is organized as follows. In Section 2, we begin with a brief review of the required equations for finite deformations of an incompressible elastic material. In Section 3, we investigate, by introducing a nonmonotonous damage softening function, the nonmonotonous behavior of experimental data when normalized uniaxial stresses and transverse vibration frequency ratios are plotted against the normalized strain intensity ratio [10,21]. In Section 4 , we have used the Ogden and Roxburgh pseudoelasticity theory to account for residual strain effects by introducing slight modifications to the model developed by Holzapfel and coworkers in [8]. In Section 5, we use the non-Gaussian average-stretch, fullnetwork model of arbitrarily oriented molecular chains [22] to derive the constitutive equations that describe the small transverse vibrations of rubber cords. A comparison of the results corresponding to simulated and experimental data is done in Section 6. Finally, in Section 7, we make some closing remarks related to our derived stress-softening model that accounts for residual strains.

\section{Theoretical Aspects}

We consider the deformation of an incompressible elastic body which in its natural configuration occupies the region $\Omega$. A material particle is assumed to be in its undeformed reference configuration of a body at the place $\mathbf{X}=X_{k} \mathbf{e}_{k}$. After a prescribed deformation, the body occupies the region $\Omega_{c}$, the current configuration, and the particle at $\mathbf{X}$ moves to the place $\mathbf{x}=\mathbf{x}_{k} \mathbf{e}_{k}$ in a common rectangular Cartesian frame $\varphi=\left\{O ; \mathbf{e}_{k}\right\}$ with origin $O$ and orthonormal basis $\mathbf{e}_{k}$. Thus, the Cauchy-Green deformation tensor $\mathbf{B} \equiv \mathbf{F F}^{T}$ has the form

$$
\mathbf{B}=\lambda_{1}^{2} \mathbf{e}_{11}+\lambda_{2}^{2} \mathbf{e}_{22}+\lambda_{3}^{2} \mathbf{e}_{33}
$$

where $\mathbf{e}_{j k} \equiv \mathbf{e}_{j} \otimes \mathbf{e}_{k}, \mathbf{e}_{i}$ are the associated orthonormal principal directions, $\mathbf{F}$ is the usual deformation gradient, and $\lambda_{i}$ denote the principal stretches in $\varphi$. Note that the magnitude of the strain intensity at a material point $\mathbf{X}$, denoted by $m$, is defined by $m \equiv \sqrt{\mathbf{B} \cdot \mathbf{B}}=\sqrt{\operatorname{tr} \mathbf{B}^{2}}$, where $\operatorname{tr}$ denotes the trace operation. In the undeformed state $\mathbf{B}=\mathbf{1}$, the identity tensor and $m=\sqrt{3}$; otherwise, $m>\sqrt{3}$ for all isochoric deformations [6]. We note that $m \geq \sqrt{3}$ for all $\lambda$, equality holding when and only when $\lambda=1$, the undeformed state. Since the principal invariants $I_{k}$ of $\mathbf{B}$ are defined by

$$
I_{1}=\operatorname{tr} \mathbf{B}, \quad I_{2}=\frac{1}{2}\left[I_{1}^{2}-\operatorname{tr}\left(\mathbf{B}^{2}\right)\right], \quad I_{3}=\operatorname{det} \mathbf{B},
$$

the magnitude of the strain intensity $m$ is given as

$$
m=\sqrt{I_{1}^{2}-2 I_{2}}
$$

\section{Characterization of the Nonmonotonous Material Behavior}

For an incompressible and isotropic elastic material, the corresponding time-independent Cauchy stress constitutive equation has the form

$$
\mathbf{T}=-p \mathbf{1}+\aleph_{1}\left(I_{1}, I_{2}\right) \mathbf{B}+\aleph_{-1}\left(I_{1}, I_{2}\right) \mathbf{B}^{-1},
$$

in which $\mathbf{T}$ is the Cauchy stress, $p$ is an undetermined pressure, and $\aleph_{\Gamma}=\aleph_{\Gamma}\left(I_{1}, I_{2}\right), \Gamma=1,-1$, denote the virgin material response functions related to the strain energy function $W=W^{*}\left(I_{1}, I_{2}\right)$, per unit reference volume, in accordance with

$$
\aleph_{1}=2 W_{1}, \quad \aleph_{-1}=-2 W_{2},
$$

wherein $W_{\alpha} \equiv \partial W^{*} / \partial I_{\alpha}$ [22]. By using (4), Elías-Zúñiga and Beatty in [7] proposed a damage type model to describe the stress-softened material behavior of the form

$$
\boldsymbol{\tau}=F(m ; M) \mathbf{T},
$$

in which $\tau$ denotes the Cauchy stress in the stress-softened material, $M$ denotes the maximum previous strain at which the material is unloaded from the primary path, and $F(m ; M)$ is an isotropic softening function at the damage level $m_{\max }=$ $M$ on the interval $m \in[\sqrt{3}, M]$. They assumed that this softening function $F(m ; M)$ is a monotone increasing function of the strain intensity that satisfies the conditions

$$
0<F(m ; M)<1, \quad F(M ; M)=1 .
$$

Based on this assumption, Elas-Zúñiga and Beatty proposed the following softening function:

$$
F(m ; M)=e^{-b \sqrt{(M-m)}},
$$

where $b$ is a dimensionless positive material softening parameter. After substituting (8) into (6), Elas-Zúñiga and Beatty obtained the following stress-softening phenomenological material model:

$$
\boldsymbol{\tau}=e^{-b \sqrt{(M-m)}} \mathbf{T} \text {. }
$$

Theoretical predictions provided by (9) were computed in $[7,10]$ and compared to experimental data for uniaxial, pure shear, and equibiaxial deformation states. There, the theoretical predictions showed reasonably good agreement with experimental data not only for the virgin loading path, but also for the reloading paths. However, in a recent publication Kazakevičiùte-Makovska [23] observed that the experimental data when plotted as the normalized stress components $\tau_{i j} / T_{i j}$ on $0<\tau_{i j} / T_{i j} \leq 1$ versus the stretch ratio $\lambda / \lambda_{\max }$ showed nonmonotonous behavior with the characteristics $S$ shaped form and she concluded that, because of the variations in shapes of the curves for different deformation cycles, different values of the softening parameters were needed to fit experimental data for a particular choice of the softening function. Moreover, she showed that the softening function 


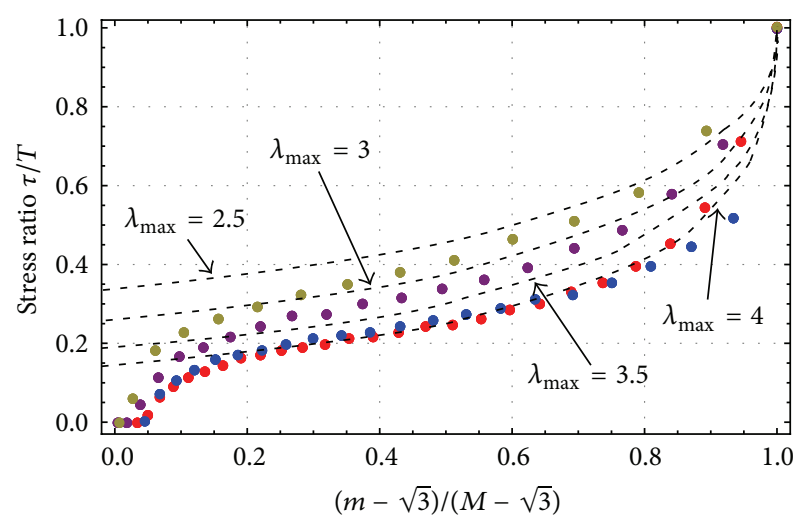

FIGURE 1: Normalized uniaxial extension data of buna-N elastomer strings plotted versus the normalized strain intensity ratio $(m-$ $\sqrt{3}) /(M-\sqrt{3})$ for which $b=0.515$ (experimental data adapted from [5]). The specimen Shore A hardness reported by the manufacturer is 50 with a density of $1.48 \mathrm{gr} / \mathrm{cm}^{3}$. Here the color dots represent the experimental data and the dashed lines represent theoretical results obtained from (8) and (9).

given by (8) fails in predicting the nonmonotonous behavior exhibited by experimental data collected by Mullins and Tobin [2], Cheng and Chen [24], and Mars and Fatemi [25] at higher stretch values. In this sense, de Tommasi et al. showed the importance of microscopic inhomogeneity to describe known experimental effects observed in amorphous materials such as the transition from diffuse to localized damage as the distribution properties are varied [26]. In fact, they showed that the monotone stress-stretch loading curve behavior is mainly due to a diffuse damage mechanism. They also considered that amorphous materials can be characterized by unstable strain domains, which gives the possibility of having homogeneous or localized damage with nonmonotone primary loading curve.

To confirm these observations, we use uniaxial tension data from a strain-controlled experiment (buna6) by Johnson on a buna-N elastomer in which, for each loading data point, there is a corresponding data point for the same amount of stretch on the unloading curve [7]. Figure 1 illustrates the normalized stress components versus the normalized strain intensity ratio $(m-\sqrt{3}) /(M-\sqrt{3})$ curves in which residual strains are considered. We can see from Figure 1 that the experimental data exhibits monotone behavior. Nevertheless, there is a wide variation between theoretical predictions provided by the corresponding uniaxial stress component given by (9) and experimental data, especially for small values of $m$ near to $\sqrt{3}$. Next, we use Elías-Zúñiga and Beatty straincontrolled experimental data on silicone elastomer cords subjected to the small amplitude transverse vibrations and plot the transverse vibrational frequency data ratio, $v_{s} / \nu=$ $\sqrt{\tau / T}$, versus the normalized strain intensity ratio, where $v$ represents the transverse vibrational frequency of the virgin string, and $v_{s}$ the corresponding frequency of the stresssoftening cord [21]. As we can see from Figure 2, the collected experimental data exhibit nonmonotone behavior. Therefore, it is clear that theoretical predictions computed from (9) must

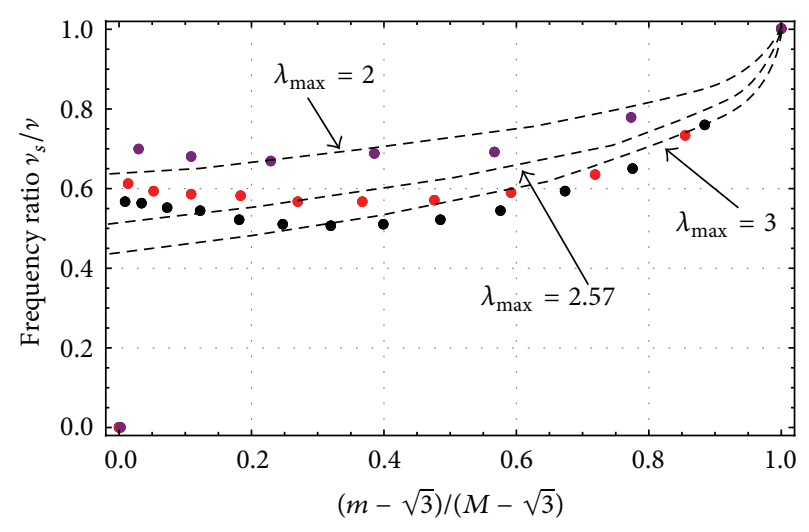

FIGURE 2: Normalized frequency ratio experimental data of silicone elastomer cords plotted versus the normalized strain intensity ratio $(m-\sqrt{3}) /(M-\sqrt{3})$ for which $b=0.6$ [21]. The specimen Shore A hardness reported by the manufacturer is 50 with a density of $1.36 \mathrm{gr} / \mathrm{cm}^{3}$. Here the color dots represent the experimental data and the dashed lines represent theoretical results obtained from (8) and (9).

deviate from experimental data since this equation considers the softening function (8) which has monotonous behavior. In Figures 1 and 2, the color dots represent experimental data and the dashed black lines are theoretical predictions. These observations and the conclusions drawn by KazakevičiūteMakovska in [23] and de Tommasi et al. in [26] motivate us to consider $F(m ; M)$ as a nonmonotone softening function that must satisfy the conditions given by (7). Therefore, we use here the nonmonotonous softening function [27]

$$
F(m ; M)=e^{-b\left[(M-m)(m / M)^{\gamma}\right]^{\alpha}},
$$

where $b$ is a positive softening material parameter and $\alpha$ and $\gamma$ are positive scaling constants chosen to best fit experimental data for a given rubber-like material. Then, the substitution of (10) into (6) yields the following material model:

$$
\boldsymbol{\tau}=\mathbf{T} e^{-b\left[(M-m)(m / M)^{\gamma}\right]^{\alpha}} .
$$

Notice that (11) does not take into account permanent set effects. Therefore, in the next section, we will use pseudoelasticity theory to obtain an expression that will include residual strain effects.

\section{Inclusion of Residual Strain Effects}

Hyperelastic materials are rheologically described by employing strain energy density functions which are related to the energy stored in the material; there are several hyperelastic material models proposed to predict elastomeric material behavior, but most of them do not take into account residual strains [4-7]. Holzapfel and coworkers proposed the following phenomenological model to include residual strain effects [8]:

$$
W_{r s}=W\left(\lambda_{1}, \lambda_{2}, \lambda_{3}\right)+\widehat{W}\left(\lambda_{a}, \xi_{a}\right)
$$


Here, $W\left(\lambda_{1}, \lambda_{2}, \lambda_{3}\right)$ represents the strain energy function associated with the primary loading path that is supplemented by a damage mechanism energy function $\widehat{W}\left(\lambda_{a}, \xi_{a}\right)$ which depends on the principal stretches $\lambda_{a}$, and the discontinuous damage variables $\xi_{a}, a=1,2,3$, which are related to the maximum principal stretch values; thus, the modified strain energy function $W_{r s}$ depends on the principal stretches. Based on this modified strain energy function $W_{r s}$, Holzapfel and coworkers derived a stress-softening material model by using the theory of pseudoelasticity developed in [4] and implemented it into a finite element program. Later, Dorfmann and Ogden proposed a model based on the theory of pseudoelasticity to capture the stress-softening and residual strain effects [28]. This model uses the monotonic dissipation energy functions $\phi_{1}$ and $\phi_{2}$ that are assumed to depend on the amount of stretch and evolve with the deformation material history. This model has the form

$$
\begin{aligned}
\widehat{W} & \left(\lambda_{1}, \lambda_{2}, \eta_{1}, \eta_{2}\right) \\
= & \eta_{1} W\left(\lambda_{1}, \lambda_{2}, \lambda_{1}^{-1} \lambda_{2}^{-1}\right)+\left(1-\eta_{2}\right) \widehat{N}\left(\lambda_{1}, \lambda_{2}\right) \\
& +\phi_{1}\left(\eta_{1}\right)+\phi_{2}\left(\eta_{2}\right),
\end{aligned}
$$

where $\widehat{N}$ is a function that characterizes the residual strains, $\eta_{1}$ is a damage or softening variable, $\eta_{2}$ represents the residual strain variable, and $W\left(\lambda_{1}, \lambda_{2}, \lambda_{1}^{-1} \lambda_{2}^{-1}\right)$ represents the material strain energy density when $\eta_{1}$ and $\eta_{2}$ are inactive.

Here, we follow Holzapfel et al. phenomenological model given by (12) and assume that the residual strain energy that accounts for the damage material mechanism has the form

$$
\widehat{W}\left(\lambda_{a}, \xi_{a}\right)=\mu \sum_{a=1}^{3}\left[\xi_{a}\left(\lambda_{\max a}^{n}-\lambda_{a}^{n}\right)+\phi\left(\xi_{a}\right)\right],
$$

where $\mu$ is the material shear modulus, $\phi$ represents the damage function, $\lambda_{\max a}, a=1,2,3$, are the maximum values of the principal stretches at which unloading begins on the primary loading path, and $n$ is a positive scaling constant that in general takes the value of one. By using the concepts of pseudo-elastic theory [4], we assume that [17]

$$
\frac{\partial \widehat{W}\left(\lambda_{a}, \xi_{a}\right)}{\partial \xi_{a}}=0 .
$$

Substitution of (14) into (15) yields

$$
\frac{\partial \widehat{W}\left(\lambda_{a}, \xi_{a}\right)}{\partial \xi_{a}}=\left(\lambda_{\max a}^{n}-\lambda_{a}^{n}\right)+\frac{\partial \phi\left(\xi_{a}\right)}{\partial \xi_{a}}=0
$$

from which

$$
-\frac{\partial \phi\left(\xi_{a}\right)}{\partial \xi_{a}}=\left(\lambda_{\max a}^{n}-\lambda_{a}^{n}\right) .
$$

If $\phi^{\prime}\left(\xi_{a}\right)$ is assumed to have the form

$$
-\frac{\partial \phi\left(\xi_{a}\right)}{\partial \xi_{a}}=C \xi_{a}
$$

then the integration of (18) provides

$$
\phi\left(\xi_{a}\right)=-\left[\frac{C}{2} \xi_{a}^{2}+d_{0}\right],
$$

where $C$ is a positive dimensionless material constant and $d_{0}$ is an integration constant. Notice from (17) and (18) that

$$
\xi_{a}=\frac{1}{C}\left(\lambda_{\max a}^{n}-\lambda_{a}^{n}\right)
$$

On the primary loading path, $\xi_{a}$ is inactive, while on the unloading path, $\xi_{a}$ has the value given by (20). Substitution of (20) into (14) gives the following pseudo-elastic residual strain energy density per unit volume:

$$
\widehat{W}\left(\lambda_{a}, \xi_{a}\right)=\frac{\mu}{C} \sum_{a=1}^{3}\left[\frac{1}{2}\left(\lambda_{\max a}^{n}-\lambda_{a}^{n}\right)^{2}-C d_{0}\right] .
$$

Therefore, the strain energy function that accounts for residual strains of an incompressible, isotropic, and hyperelastic material, in accordance with pseudo-elastic theory, has the form

$$
W_{r s}=W\left(\lambda_{1}, \lambda_{2}, \lambda_{3}\right)+\frac{\mu}{C} \sum_{a=1}^{3}\left[\frac{1}{2}\left(\lambda_{\max a}^{n}-\lambda_{a}^{n}\right)^{2}-C d_{0}\right] .
$$

In the next section, we will use (22) to derive a material model that will be used to characterize experimental data of elastomeric rubber cords subjected to small transverse vibrations.

\section{A Nonmonotonous Material Model}

To illustrate the application of the softening function given by (10) as well as of the residual strain energy function (22), we first consider the Arruda-Boyce constitutive equation for an average-stretch, full-network of arbitrarily oriented molecular chains and modify this to include stress-softening and residual strain effects. Then, we will use Taylors' equation to derive the material model that describes the dynamics response of rubber-like cords when subjected to small amplitude transverse vibrations.

First, let us begin with the modification of the averagestretch, full-network material model to include residual strain effects. For this material model, it is well known that the strain energy per unit volume for the loading path is given by

$$
W\left(\lambda_{1}, \lambda_{2}, \lambda_{3}\right)=\mu N\left(\beta \lambda_{r}+\ln \left(\frac{\beta}{\sinh \beta}\right)\right)-c_{8},
$$

where $\lambda_{r}$ is the relative chain stretch defined by

$$
\lambda_{r}=\frac{\lambda_{\text {chain }}}{\lambda_{L}}
$$

and $\lambda_{L}=\sqrt{N}$ represents the fully extended chain stretch, $N$ is the chain number of rigid links, each of length $l$, and 
$\lambda_{\text {chain }}$ is the chain deformation that in the affine deformation is determined by

$$
\lambda_{\text {chain }} \equiv \sqrt{\frac{I_{1}}{3}}
$$

$\beta \equiv \mathscr{L}^{-1}\left(\lambda_{r}\right)$ is the inverse of the Langevin function $\mathscr{L}(\beta)$ which is defined as

$$
\lambda_{r}=\mathscr{L}(\beta) \equiv \operatorname{coth} \beta-\frac{1}{\beta}
$$

and $c_{8}$ is a constant that ensures that the strain energy vanishes in the undeformed state [22, 27]. Substitution of (23) into (22) provides the modified non-Gaussian pseudostrain energy per unit volume that accounts for residual strains on the unloading path; that is,

$$
\begin{aligned}
W_{r s}= & \mu N\left(\beta \lambda_{r}+\ln \left(\frac{\beta}{\sinh \beta}\right)\right) \\
& +\frac{\mu}{C} \sum_{a=1}^{3}\left[\frac{1}{2}\left(\lambda_{\max a}^{n}-\lambda_{a}^{n}\right)^{2}\right]+D,
\end{aligned}
$$

where $D$ is an energy constant. This is the strain energy density of an elastic material for which $\xi_{a}$ is active.

Substituting of (23) into (4) and with the aid of (5), the Cauchy stress-stretch averaging network model components for the virgin material, for which $\xi_{a}=0$, becomes

$$
T_{k}=-p+\frac{\mu \beta}{3 \lambda_{r}} \lambda_{k}^{2}
$$

Eliminating the pressure from (28) gives

$$
T_{j}-T_{k}=\frac{\mu \beta}{3 \lambda_{r}}\left(\lambda_{j}^{2}-\lambda_{k}^{2}\right)
$$

where $j \neq k=1,2,3$ (no sum). The Cauchy stress-stretch constitutive equation for a stress-softening material can be obtained by substituting (27) into (4) and by using (5) and (11). This yields the following stress-softening components:

$$
\begin{array}{r}
\tau_{k}=\left[-p+\frac{\mu \beta}{3 \lambda_{r}} \lambda_{k}^{2}\right. \\
\left.+\frac{\mu \lambda_{k}}{2 C} f_{k}\left(\lambda_{1}, \lambda_{2}, \lambda_{3}\right)\right] e^{-b \sqrt{(M-m)(m / M)}}, \\
k=1,2,3 \text { (no sum), }
\end{array}
$$

where

$$
f_{k}\left(\lambda_{1}, \lambda_{2}, \lambda_{3}\right)=\frac{\partial \sum_{a=1}^{3}\left(\lambda_{\max a}^{n}-\lambda_{a}^{n}\right)^{2}}{\partial \lambda_{k}} .
$$

Then, on elimination of $p$ from (30), we get that

$$
\begin{aligned}
\tau_{j}-\tau_{k} & \\
= & {\left[\frac{\mu \beta}{3 \lambda_{r}}\left(\lambda_{j}^{2}-\lambda_{k}^{2}\right)+\frac{\mu}{2 C}\right.} \\
& \left.\quad \times\left(\lambda_{j} f_{j}\left(\lambda_{1}, \lambda_{2}, \lambda_{3}\right)-\lambda_{k} f_{k}\left(\lambda_{1}, \lambda_{2}, \lambda_{3}\right)\right)\right] \\
& \times e^{-b \sqrt{(M-m)(m / M)}},
\end{aligned}
$$

where, in general, $j \neq k=1,2,3$ (no sum).

Recalling that for an incompressible material, the engineering stress $\boldsymbol{\sigma}$ is related to the Cauchy stress by

$$
\sigma=\mathrm{TF}^{-1}
$$

then, the uniaxial engineering stress-stretch relation for an average-stretch, full-network stress-softening material model that considers residual strain effects is obtained by using (31), (32), and (33):

$$
\begin{aligned}
\sigma_{s}= & \frac{\mu \beta}{3 \lambda_{r}}\left(\lambda-\lambda^{-2}\right)+\frac{\mu}{C} \\
& \left.\times\left(-n \lambda^{(n-1)}\left(\lambda_{\max }^{n}-\lambda^{n}\right)+n \lambda^{-(1+n / 2)}\left(\lambda_{\max }^{-n / 2}-\lambda^{-n / 2}\right)\right)\right] \\
& \times e^{-b \sqrt{(M-m)(m / M)}} .
\end{aligned}
$$

Here,

$$
m=\sqrt{\lambda^{4}+2 \lambda^{-2}}
$$

and the relative chain stretch which can be obtained from (24) and (25) is given as

$$
\lambda_{r}=\sqrt{\frac{1}{3 N}\left(\lambda^{2}+2 \lambda^{-1}\right)} .
$$

In equibiaxial extension, the engineering stress-softened relation is given by [27]

$$
\begin{aligned}
\sigma_{s}= & {\left[\frac{\mu \beta}{3 \lambda_{r}}\left(\lambda-\lambda^{-5}\right)+\frac{\mu}{C}\right.} \\
& \left.\times\left(-n \lambda^{(n-1)}\left(\lambda_{\max }^{n}-\lambda^{n}\right)+n \lambda^{-(1+2 n)}\left(\lambda_{\max }^{-2 n}-\lambda^{-2 n}\right)\right)\right] \\
& \times e^{-b \sqrt{(M-m)(m / M)}},
\end{aligned}
$$

where

$$
\lambda_{r}=\sqrt{\frac{1}{3 N}\left(2 \lambda^{2}+\lambda^{-4}\right)}, \quad m=\sqrt{2 \lambda^{4}+\lambda^{-8}} .
$$


In the case of a pure shear or plane strain compression deformation state, the engineering stress-softened constitutive equation is given by

$$
\begin{aligned}
\sigma_{s}= & \frac{\mu \beta}{3 \lambda_{r}}\left(\lambda-\lambda^{-3}\right)+\frac{\mu}{C} \\
& \left.\times\left(-n \lambda^{(n-1)}\left(\lambda_{\max }^{n}-\lambda^{n}\right)+n \lambda^{-(1+n)}\left(\lambda_{\max }^{-n}-\lambda^{-n}\right)\right)\right] \\
& \times e^{-b \sqrt{(M-m)(m / M)}},
\end{aligned}
$$

where

$$
\lambda_{r}=\sqrt{\frac{1}{3 N}\left(\lambda^{2}+\lambda^{-2}+1\right)}, \quad m=\sqrt{\lambda^{4}+\lambda^{-4}+1}
$$

We next use Taylors' equation [21] to derive the nonmonotonous material constitutive equation that describes the behavior of rubber cords subjected to transverse vibrations.

5.1. Transverse Vibration of Elastomeric Cords. Here, we briefly review some of the fundamental concepts that are used to describe the small amplitude transverse vibrations of rubberlike cords. Full details of the theoretical analysis can be found in [21].

5.1.1. Basic Equations. The frequency of the fundamental mode of the small amplitude transverse vibrations about the stretched equilibrium position of an elastomeric cord with fixed ends is given by Taylor's equation as

$$
v=v_{0} \sqrt{\frac{T}{\mu \lambda^{2}}}, \quad v_{0} \equiv \sqrt{\frac{A_{0} \mu}{4 l_{0}^{2} \rho_{0}}},
$$

where $l_{0}$ is the initial cord length, $A_{0}$ represents the uniform cross-sectional area, $\rho_{0}$ is the mass density per unit length, and $T$ is the Cauchy stress for the uniaxial loading of the virgin material given by (29). In the case of the stresssoftening material, the transverse vibrations frequency $v_{s}$ can be determined by using the following equation:

$$
v_{s}=v_{0} \sqrt{\frac{\tau}{\mu \lambda^{2}}},
$$

where $\tau$ represents the stress-softening Cauchy stress described by (32). Notice from previous remarks that $\nu / \nu_{s}=$ $\sqrt{T / \tau} \geq 1$. Therefore, the magnitude of the transverse vibrations frequency of the stress-softening material cord is always less than the corresponding frequency of the virgin material at each value of the strain intensity $m \in(\sqrt{3}, M)$, equality holding when and only when $m=M$. We now introduce the normalized small amplitude transverse vibration frequencies for the virgin and stress-softened materials: $f=v / \nu_{0}$ and $f_{s}=\nu_{s} / \nu_{0}$. The corresponding constitutive equation of $f_{s}$, for the softening material, can be obtained by substituting (34) into (42); this yields

$$
\begin{aligned}
& f_{s} \\
& =\left[\left\{\frac{\beta}{3 \lambda_{r}}\left(1-\lambda^{-3}\right)+\frac{1}{C}\right.\right. \\
& \left.\quad \times\left(-n \lambda^{(n-2)}\left(\lambda_{\max }^{n}-\lambda^{n}\right)+n \lambda^{-(2+n / 2)}\left(\lambda_{\max }^{-n / 2}-\lambda^{-n / 2}\right)\right)\right\} \\
& \left.\quad \times e^{-b \sqrt{(M-m)(m / M)}}\right]^{1 / 2} .
\end{aligned}
$$

Of course, the normalized virgin cord frequency $f$ is obtained from (43) with $m=M$ and $\lambda=\lambda_{\max }$.

\section{Theoretical Simulations}

To assess the precision of the derived constitutive (43) which includes residual strains and has a nonmonotonous softening function that describes Mullins effect, we use experimental data collected during the small amplitude transverse vibrations of buna-N, silicone, and neoprene elastomers [21]. Notice that only four constitutive material constants and one fitting parameter are required to use (43), that is, the shear modulus $\mu$, the chain number of links $N$, the stresssoftening parameter $b$, the residual strain material constant $C$, and the fitting parameter $n$. In what follows, we will show that the value of $n=1$ for the normalized transverse vibration frequency model described in (43) provides good fit to experimental data.

We begin by using data collected from the transverse vibrations of a buna-N elastomer material cord [21]. Figure 3 illustrates the predicted curves obtained from (43) compare to experimental data. Notice from Figure 3(a) that the normalized transverse frequency predictions are in good agreement with experimental data for the several loading and unloading cycles that exhibit residual strains. The nonmonotonous characteristic behavior of the buna- $\mathrm{N}$ experimental data is shown in Figure 3(b) where the ratio of the transverse vibrations frequency of the softening and the virgin material is plotted versus the normalized strain intensity ratio $(m-\sqrt{3}) /(M-\sqrt{3})$. One can notice that there is some discrepancies between experimental data and theoretical predictions that could be due to viscoelastic material effects that were neglected during the derivation of the material model (43). In this case, the constitutive material constants used to best fit experimental data are $\nu_{0}=90 \mathrm{~Hz}$, $N=20.89, b=0.61$, and $C=4 \mathrm{MPa}$.

Figures 4 and 5 illustrate data of stress softening collected from silicone and neoprene rubber strings, respectively. We can see that predicted curves, for the proposed material model defined by (43), stand in good agreement with experimental data for the several loading and unloading cycles. Moreover, the nonmonotonous behavior and residual strains depicted in Figures 4(b) and 5(b) are described well by our derived material model. In these figures, the dots represent 


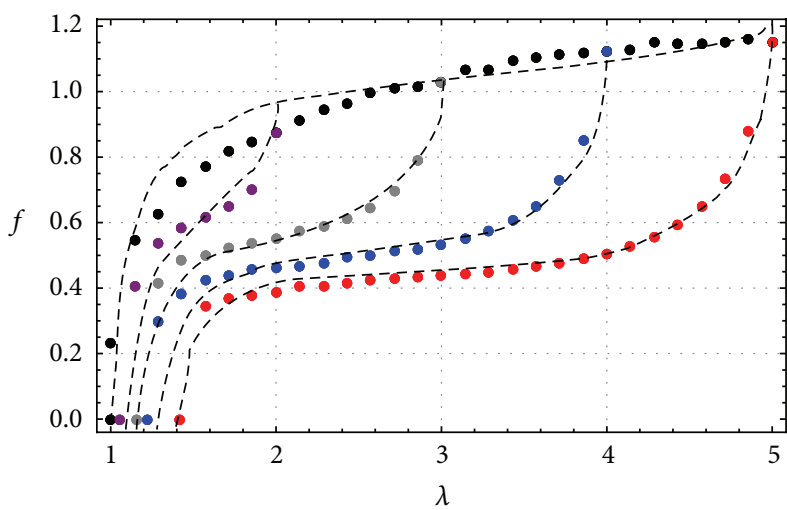

(a)

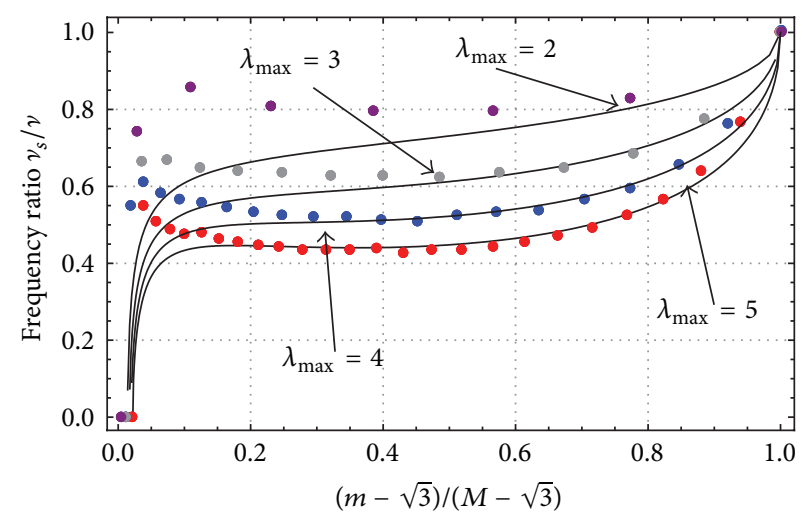

(b)

FIgURE 3: Comparison of buna-N rubber strings experimental data with theoretical predictions computed from the average-stretch, fullnetwork model for which $v_{0}=90 \mathrm{~Hz}, n=1, N=20.89, b=0.61$, and $C=4 \mathrm{MPa}$. (a) Normalized frequency ratio $f$ versus stretch; (b) normalized frequency ratio $f$ versus the normalized strain intensity ratio $(m-\sqrt{3}) /(M-\sqrt{3})$ (experimental data adapted from [21]). The specimen Shore A hardness reported by the manufacturer is 60 with a density of $1.19 \mathrm{gr} / \mathrm{cm}^{3}$. Here, the color dots represent the experimental data and the solid and dashed lines represent theoretical results obtained from (29) and (43).

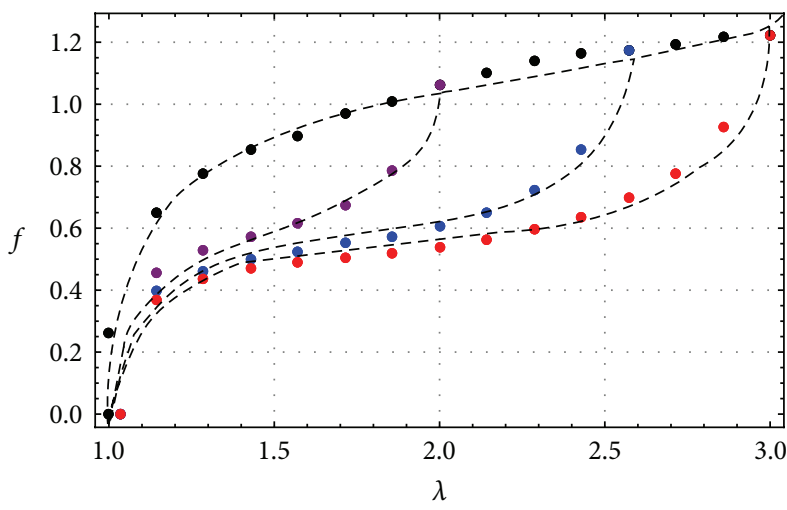

(a)

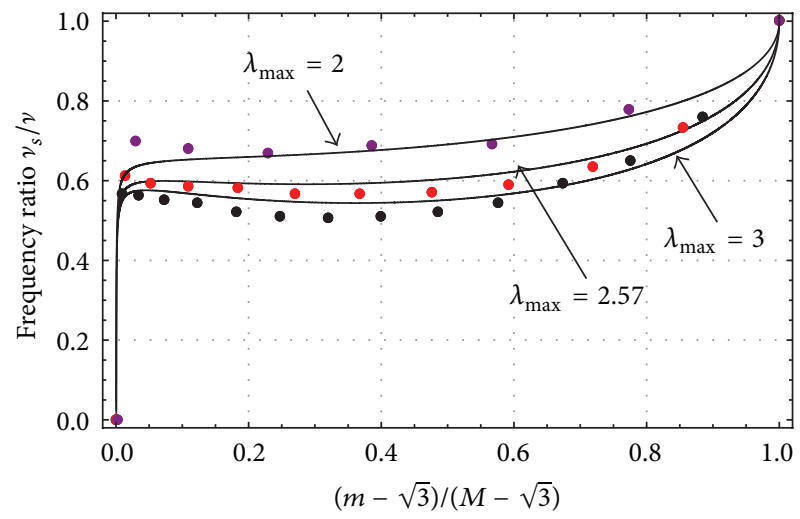

(b)

FIGURE 4: Comparison of silicone rubber strings experimental data with theoretical predictions computed from the average-stretch, fullnetwork model for which $\nu_{0}=89 \mathrm{~Hz}, n=1, N=6.3, b=0.8$, and $C=24 \mathrm{MPa}$. (a) Normalized frequency ratio $f$ versus stretch; (b) normalized frequency ratio $f$ versus the normalized strain intensity ratio $(m-\sqrt{3}) /(M-\sqrt{3})$. The specimen Shore A hardness reported by the manufacturer is 50 with a density of $1.36 \mathrm{gr} / \mathrm{cm}^{3}$ (experimental data adapted from [21]). Here, the color dots represent the experimental data and the solid and dashed lines represent theoretical results obtained from (29) and (43).

the experimental data and the solid and dashed lines represent theoretical results obtained, respectively, from (29) and (43). The material constants for the material model are provided by a best fit analysis and these are listed in the figure captions.

\section{Conclusions}

In this paper, we have used a nonmonotonous softening function that accounts for the microstructural material damage upon deformation from the natural, undistorted state of the virgin material. This phenomenological softening function is based on three parameters; one is a positive softening material parameter and the other two are basically positive scaling constants that in general have the values of $\alpha=1 / 2$ and $\gamma=1$. We have shown that this softening function predicts well the nonmonotonous behavior observed in rubber-like materials when experimental data are plotted against the normalized strain intensity ratio. Nevertheless, there is a variation in the results for values of $m$ near to $\sqrt{3}$, which we believe is due to some viscoelastic and anisotropic effects.

The Arruda-Boyce constitutive equation for an averagestretch, full-network model of arbitrarily oriented molecular chains was modified to include the residual strain effect for the unloading paths as described by the simple constitutive relation (30). Then, we have used Taylors' equation and derived the constitutive equation that describes the small transverse vibrations of elastomeric cords subjected to uniaxial extension. This model captures well the qualitative and quantitative behavior shown by experimental data collected from samples of buna-N, silicone, and neoprene elastomeric cords. In modeling the small transverse vibrations of the aforementioned rubber cords, we have only used four material constants: $v_{0}$, the chain number of rigid links $N$, 


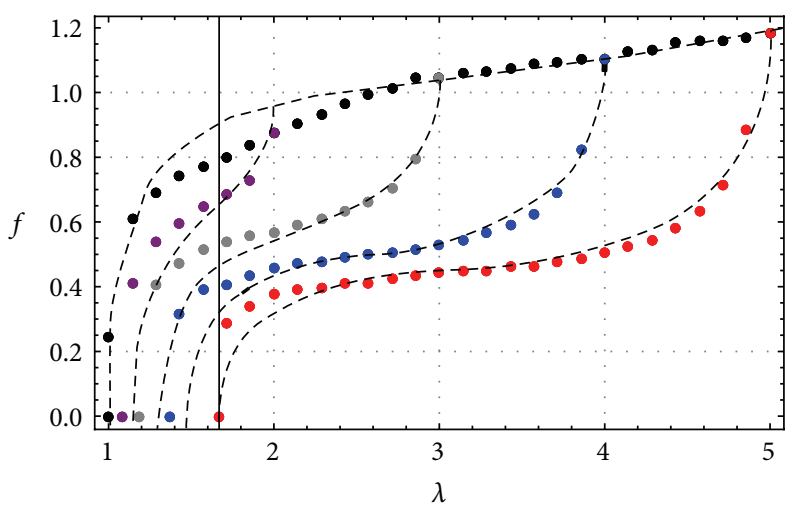

(a)

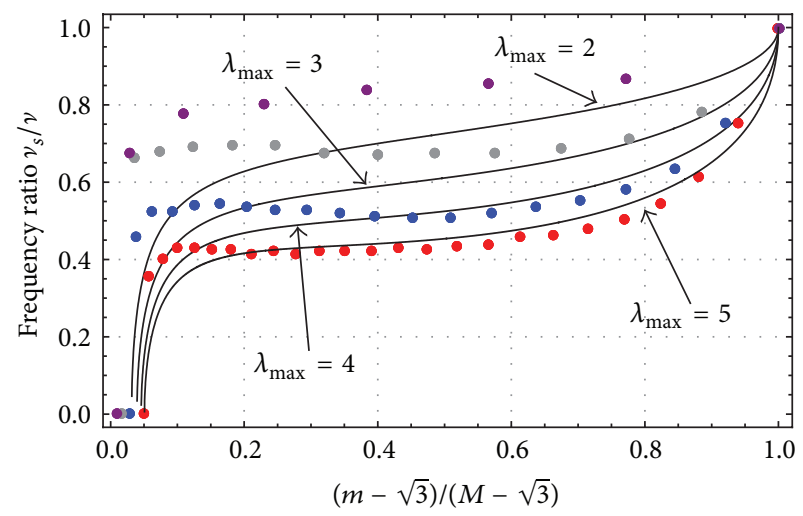

(b)

FIGURE 5: Comparison of neoprene rubber strings experimental data with theoretical predictions computed from the average-stretch, fullnetwork model for which $v_{0}=69 \mathrm{~Hz}, n=1, N=19.95, b=0.58$, and $C=2.5 \mathrm{MPa}$. (a) Normalized frequency ratio $f$ versus stretch; (b) normalized frequency ratio $f$ versus the normalized strain intensity ratio $(m-\sqrt{3}) /(M-\sqrt{3})$. The specimen Shore A hardness reported by the manufacturer is 40 with a density of $1.17 \mathrm{gr} / \mathrm{cm}^{3}$ (experimental data adapted from [21]). Here, the color dots represent the experimental data and the solid and dashed lines represent theoretical results obtained from (29) and (43).

the material softening parameter $b$, and a positive material constant $C$ that is related to the pseudoelastic residual strain energy. Based on the accuracy of our proposed nonmonotonous model (43) to predict experimental data, we can conclude that the extent of damage of rubber-like materials, when subjected to small transverse vibrations, can be conveniently determined by considering the nonmonotonous behavior exhibited by experimental data. Furthermore, our proposed damage material mechanism model given by (14) can be applied to three-dimensional deformation states since the damage function is controlled by the strain energy associated with the primary deformation and not just by the specific deformation from which it is calculated.

Finally, we have also found, by using our theoretical model, excellent agreement with stress-softened experimental data collected for other deformation states. The results of this new work will be reported in a subsequent paper.

\section{Acknowledgment}

This work was funded by Tecnológico de Monterrey-Campus Monterrey, through the Research Chair in Nanomaterials for Medical Devices and Research Chair in Intelligent Machines and from Consejo Nacional de Ciencia y Tecnología (Conacyt), México.

\section{References}

[1] L. J. Mullins, "Effect of stretching on the properties of rubber," Journal of Rubber Research, vol. 16, pp. 275-289, 1947.

[2] L. Mullins and N. R. Tobin, "Theoretical model for the elastic behavior of filledreinforced vulcanized rubbers," Journal of Rubber Chemestry and Technology, vol. 30, no. 2, pp. 555-571, 1957.

[3] S. Govindjee and J. Simo, "A micro-mechanically based continuum damage model for carbon black-filled rubbers incorporating Mullins' effect," Journal of the Mechanics and Physics of Solids, vol. 39, no. 1, pp. 87-112, 1991.
[4] R. W. Ogden and D. G. Roxburgh, "A pseudo-elastic model for the Mullins effect in filled rubber," Proceedings of the Royal Society A, vol. 455, no. 1988, pp. 2861-2877, 1999.

[5] M. A. Johnson and M. F. Beatty, “The Mullins effect in uniaxial extension and its influence on the transverse vibration of a rubber string," Continuum Mechanics and Thermodynamics, vol. 5, no. 2, pp. 83-115, 1993.

[6] M. F. Beatty and S. Krishnaswamy, "A theory of stress-softening in incompressible isotropic materials," Journal of the Mechanics and Physics of Solids, vol. 48, no. 9, pp. 1931-1965, 2000.

[7] A. Elías-Zúñiga and M. F. Beatty, "A new phenomenological model for stress-softening in elastomers," Zeitschrift fur Angewandte Mathematik und Physik, vol. 53, no. 5, pp. 794-814, 2002.

[8] G. A. Holzapfel, M. Stadler, and R. W. Ogden, "Aspects of stress softening in filled rubbers incorporating residual strains," in Proceedings of the First European Conference on Constitutive Models for Rubber, A. Dorfmann and A. Muhr, Eds., pp. 189193, Balkema, Rotterdam, The Netherlands, 1999.

[9] E. A. de Souza Neto, D. Perić, and D. R. J. Owen, "A phenomenological three-dimensional rate-idependent continuum damage model for highly filled polymers: formulation and computational aspects," Journal of the Mechanics and Physics of Solids, vol. 42, no. 10, pp. 1533-1550, 1994.

[10] A. Elías-Zúñiga, "A phenomenological energy-based model to characterize stress-softening effect in elastomers," Polymer, vol. 46, no. 10, pp. 3496-3506, 2005.

[11] A. Dorfmann and R. W. Ogden, "A constitutive model for the Mullins effect with permanent set in particle-reinforced rubber," International Journal of Solids and Structures, vol. 41, no. 7, pp. 1855-1878, 2004.

[12] G. Marckmann, E. Verron, L. Gornet, G. Chagnon, P. Charrier, and P. Fort, "A theory of network alteration for the Mullins effect," Journal of the Mechanics and Physics of Solids, vol. 50, no. 9, pp. 2011-2028, 2002.

[13] J. Diani, M. Brieu, and J. M. Vacherand, "A damage directional constitutive model for Mullins effect with permanent set and induced anisotropy," European Journal of Mechanics, vol. 25, no. 3, pp. 483-496, 2006. 
[14] R. Kazakevičiūtè-Makovska and R. Kačianauskas, "Modelling of stress softening in elastomeric materials," Mechanics Research Communications, vol. 31, no. 4, pp. 395-403, 2004.

[15] D. de Tommasi, G. Puglisi, and G. Saccomandi, "A micromechanics-based model for the Mullins effect," Journal of Rheology, vol. 50, no. 4, pp. 495-512, 2006.

[16] S. R. Rickaby and N. H. Scott, "A cyclic stress softening model for the Mullins effect," International Journal of Solids and Structures, vol. 50, pp. 111-120, 2013.

[17] R. Córdova, A. Elías-Zúñiga, L. Elizalde et al., "Polymeric materials reinforced with multiwall carbon nanotubes: a constitutive material model," Materials, vol. 6, no. 7, pp. 2873-2891, 2013.

[18] P. D’Ambrosio, D. de Tommasi, D. Ferri, and G. Puglisi, "A phenomenological model for healing and hysteresis in rubberlike materials," International Journal of Engineering Science, vol. 46, no. 4, pp. 293-305, 2008.

[19] D. de Tommasi, S. Marzano, G. Puglisi, and G. Zurlo, "Damage and healing effects in rubber-like balloons," International Journal of Solids and Structures, vol. 46, no. 22-23, pp. 3999-4005, 2009.

[20] S. Toki, I. Sics, S. Ran et al., "New insights into structural development in natural rubber during uniaxial deformation by in situ synchrotron X-ray diffraction," Macromolecules, vol. 35, no. 17, pp. 6578-6584, 2002.

[21] A. Elías-Zúñiga and M. F. Beatty, "Stress-softening effects in the transverse vibration of a non-Gaussian rubber string," Meccanica, vol. 38, no. 4, pp. 419-433, 2003.

[22] M. F. Beatty, "An average-stretch full-network model for rubber elasticity," Journal of Elasticity, vol. 70, no. 1-3, pp. 65-86, 2003.

[23] R. Kazakevičiūtè-Makovska, "Experimentally determined properties of softening functions in pseudo-elastic models of the Mullins effect," International Journal of Solids and Structures, vol. 44, no. 11-12, pp. 4145-4157, 2007.

[24] M. Cheng and W. Chen, "Experimental investigation of the stress-stretch behavior of EPDM rubber with loading rate effects," International Journal of Solids, vol. 40, no. 18, pp. 47494768, 2003.

[25] W. V. Mars and A. Fatemi, "Observations of the constitutive response and characterization of filled natural rubber under monotonic and cyclic multiaxial stress states," Journal of Engineering Materials and Technology, vol. 126, no. 1, pp. 19-28, 2004.

[26] D. de Tommasi, G. Puglisi, and G. Saccomandi, "Localized versus diffuse damage in amorphous materials," Physical Review Letters, vol. 100, no. 8, Article ID 085502, 4 pages, 2008.

[27] A. Elías-Zúñiga and C. A. Rodríguez, "A non-monotonous damage function to characterize stress-softening effects with permanent set during inflation and deflation of rubber balloons," International Journal of Engineering Science, vol. 48, no. 12, pp. 1937-1943, 2010.

[28] A. Dorfmann and R. W. Ogden, "A pseudo-elastic model for loading, partial unloading and reloading of particle-reinforced rubber," International Journal of Solids and Structures, vol. 40, no. 11, pp. 2699-2714, 2003. 

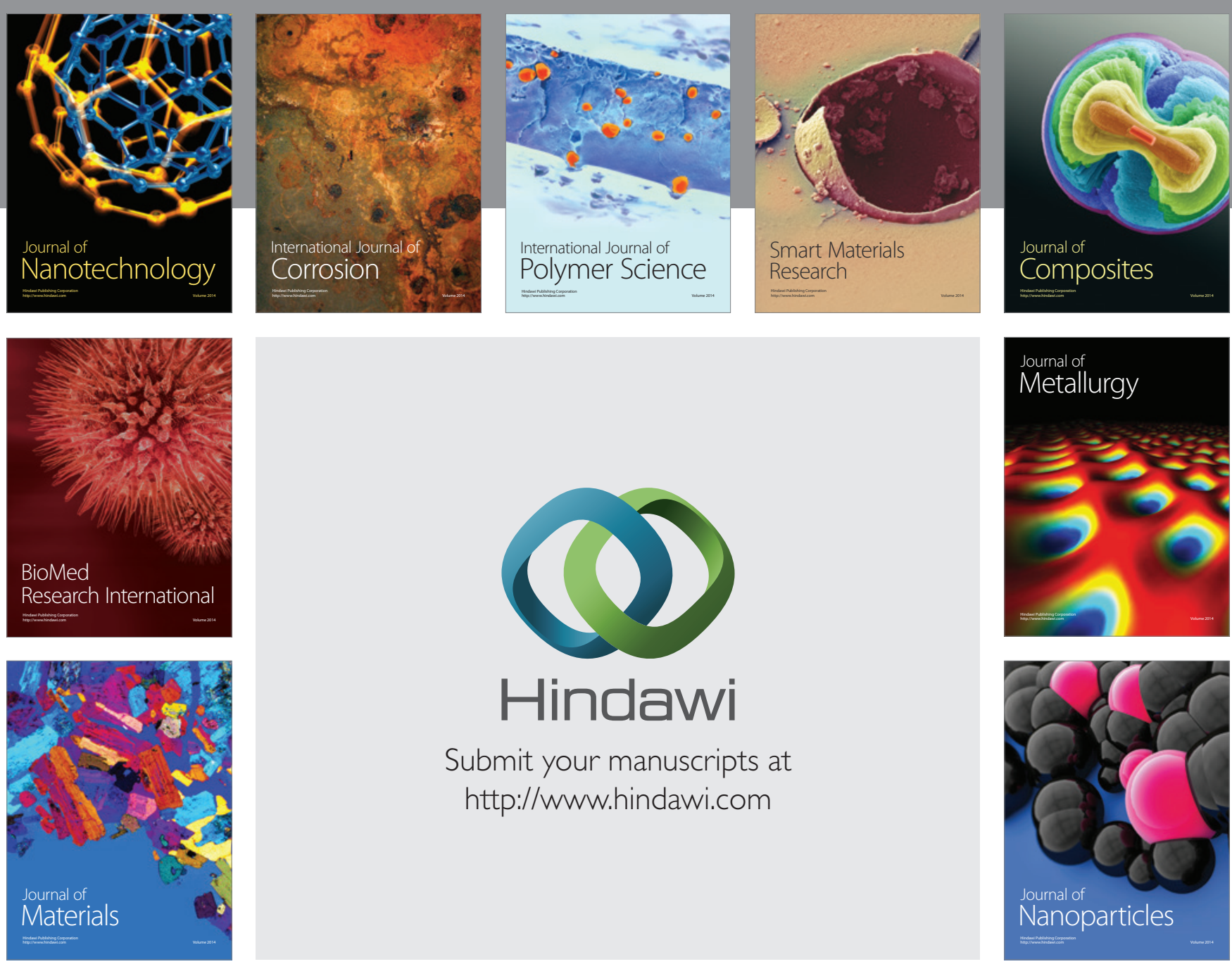

Submit your manuscripts at http://www.hindawi.com
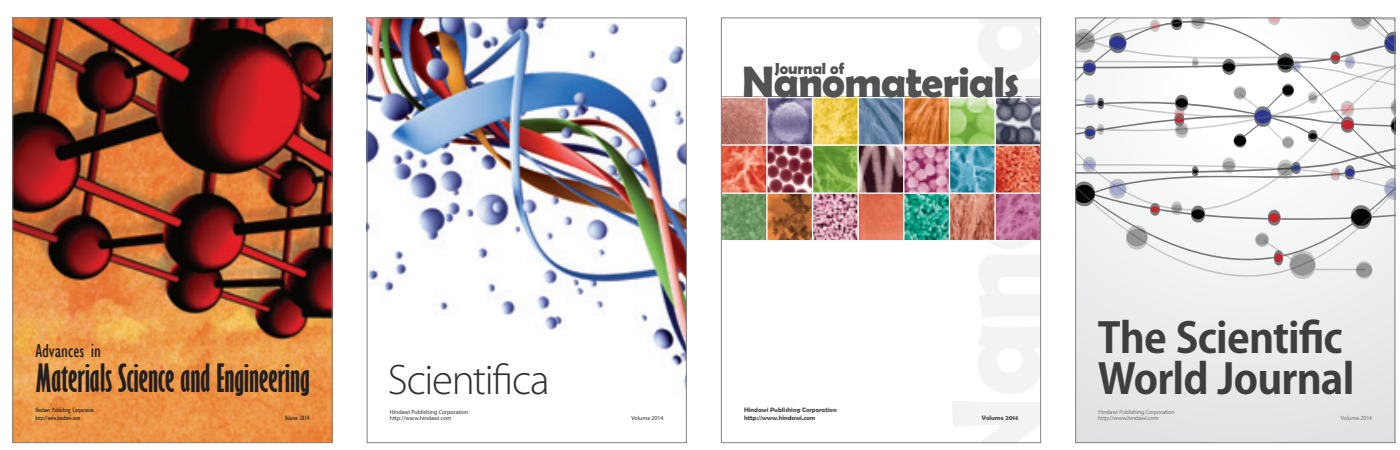

\section{The Scientific World Journal}
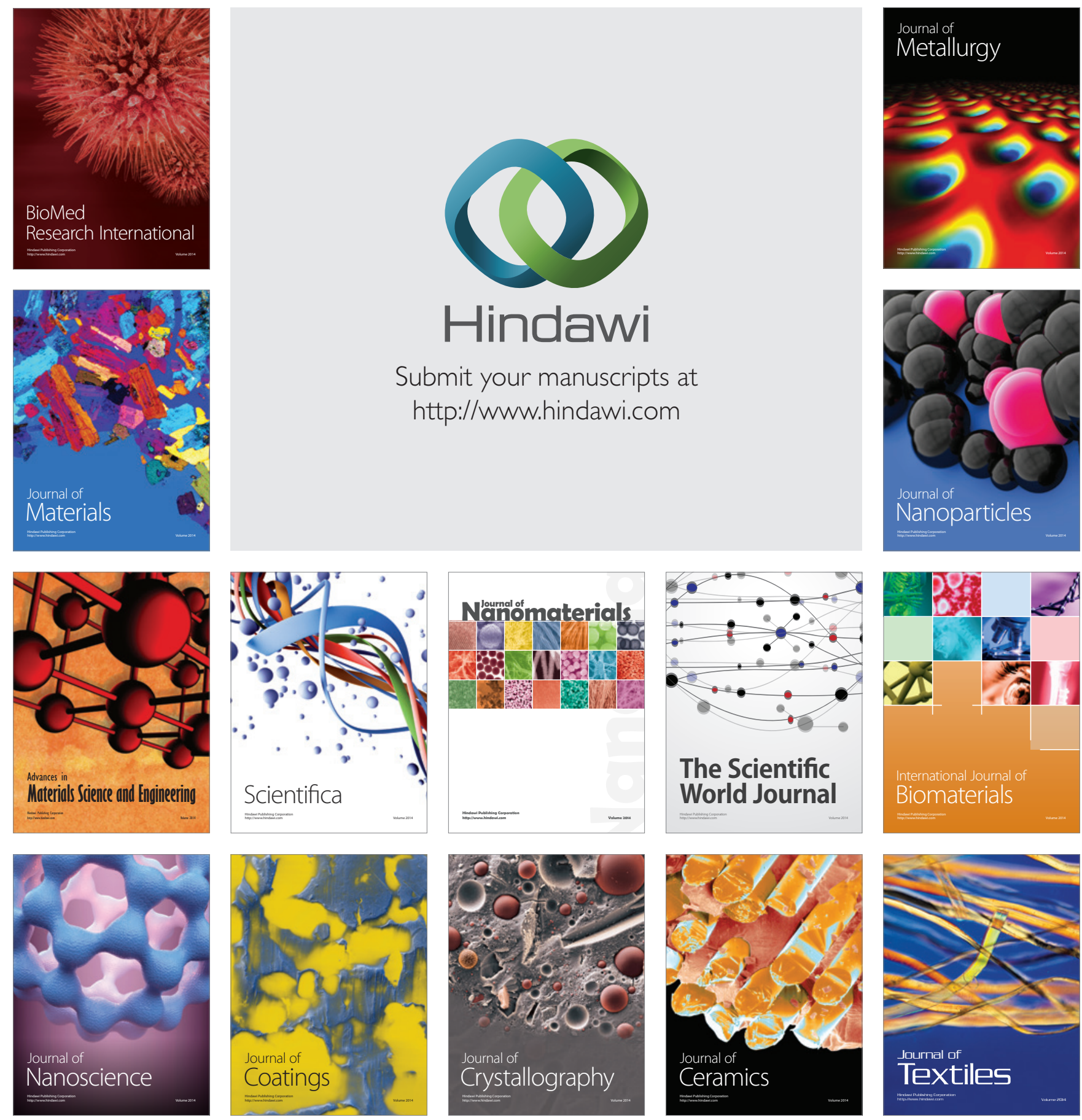